\section{INFORMATION RETRIEVAL: Program for personalized article retrieval system}

\author{
MYRON E. HATCHER \\ The Johns Hopkins School of Hygiene and Public Health \\ Baltimore, Maryland 21205 \\ THOMAS W. OAKES \\ Department of Sociology, Northern Arizona University \\ Flagstaff, Arizona 86001 \\ and \\ EDWARD KAISER \\ Technical Assistance \\ Western Bancorp Data Processing Company, P.O. Box 666 \\ Mesa, Arizona 85201
}

The objective of the program is to allow retrieval of stored information concerning articles (i.e., periodicals, monographs, books, etc.) by an individual from his own data base or a joint information set. A user of the program may retrieve articles relevant to his topic at any given time. Information is stored under descriptor words which also serve as keys to retrieval. Articles retrieved are presented alphabetically by author and under a separate listing for each descriptor. The descriptors are keys at two levels of specificity: The first level represents a general topic heading and the second level represents subtopics. An example would be "computer science" as the general topic heading, with "access control," "privacy," and "systems design" as the subtopics. The retrieval procedure is the indication of descriptors. Article information on one or more of the given descriptors is retrieved.

Input. The descriptors and other data about the articles are punched on cards and read into files. The files are used to establish the data base. Attempts to read in duplicate information or keys will result in an error message. The retrieval request is a batch process where the descriptors are listed in numeric form and the cards provide a back up system. The descriptors must conform to the general structure, but they are completely user defined.

The information on each article is divided into fixed data and variable data.

Fixed data: (1) unique number, (2) authors, (3) title, (4) periodical, (5) date published, (6) length, (7) volume and page.

Variable data: (1) abstract number assigned, (2) descriptors.

Output. The output for a request includes the descriptors. A listing for articles which match each descriptor, containing the articles information and article descriptors, is given alphabetically by article number. A listing of all articles and all descriptors is also available.

Computer and Programming Language. The system is implemented on the Xerox Sigma 6 computer. The data structure is defined in Xerox Data Management System and programs are written in Xerox COBOL.

Availability. A more detailed description and listing of this program can be obtained from Myron Hatcher, Box 376, School of Hygiene and Public Health, The Johns Hopkins University, 615 North Wolfe Street, Baltimore, Maryland 21205, at a cost of $\$ 5$.

\title{
ERRATUM
}

Fridlund, A. J., and Leberer, M. R. An inexpensive integrated circuit intracranial stimulator. Behavior Research Methods \& Instrumentation, 1976, 8(1), 21-23. Page 21, column 2, paragraph 2, the last sentence should read: "Since the meter is configured within a full-wave bridge, current read from the meter directly indicates the current being delivered to the electrode site." Page 22: The 0- to $100-\mathrm{mA}$ meter shown in the schematic diagram should be replaced directly with a 0 - to $500-\mathrm{mA}$ movement, thus allowing for indication of the higher currents sometimes required to support self-stimulation. 